ArTículo

\title{
Ya estuvo: límite de una acción
}

Ya estuvo: the limits of an action

Sandra Arteaga Santos

Universidad Autónoma de Querétaro

sandra.arteaga.santos@gmail.com

Ricardo Maldonado Soto

Universidad Nacional Autónoma de México

ricamaso@gmail.com

Original recibido: 2018/02/22

Dictamen enviado al autor: 2018/10/10

Aceptado: 2018/11/10

\section{Abstract}

Among the high number of lexicalized collocations in Mexican Spanish, an important portion of them is built with the adverb ya: ya mero 'it's coming', ya valió 'it's gone', etc. This work examines the content of one of these constructions: ya estuvo.

(1) Hoy termino con Cablevisión. Ya estuvo bonito de tanto dolor. (Twitter)

(2) Concreta, manito, tú sabes, que arrastre y que agarre, uno que pegue y ya estuvo (CREA)

(3) Ya estuvo que no superaré esta canción. (Twitter)

Based on the tenets of Cognitive Grammar (Langacker 1987: 11-30), we show that the nuclear features of the construction ya estuvo determine the existence 
of a set of construction-extensions that share the notion of "boundary". Given the nature of this construction, the study focuses exclusively on examples from Mexican Spanish. We propose that the construction ya estuvo is fully compositional since the addition of the meanings of its basic components add up to a resultative meaning. Although the construction has become an idiom, each component imposing a limit and an end point of a process can be identified; i. e., verbal telicity, extreme demarcation with adverbs of gradation, punctual demarcation by "conceptual anchor" ya (Maldonado y Delbecque 2009: 191; Delbecque y Maldonado 2011: 74) combine to depict events that are seen as fully done even when they may have not been concluded. The high level of lexicalization of ya estuvo has allowed for the construction to erase some of the closing markers, without losing the meaning of boundary. Hence the most extended form of this construction is the one which presents only the perfect verb along with the anchor ya: ya estuvo. Moreover, the presentative construction ya estuvo que + completive clause presents as relevant for the time of speech events that are about to happen. We show that the anchor $y a$ validates the notion of "end" as relevant for the time of speech and subjectively brings it closer to the conceptualizer. The remarkable relationship between the different constructions (ya estuvo bueno 'it's enough', ya estuvo que ' $\mathrm{X}$ will happen for sure', etc.) suggests that the nuclear features of the construction ya estuvo determine the constructional extensions with readings associated with concluding or stopping an event as the situation is brought to some limit or some extreme.

Key words: telicity, anchor, syntactic metonymy, terminating constructions

\section{Resumen}

Dentro del alto número de colocaciones lexicalizadas en el español mexicano, una porción importante de ellas se construye con el adverbio ya: ya mero, ya valió, etc. Este trabajo examina el contenido de una de estas construcciones: ya estuvo.

(1) Hoy termino con Cablevisión. Ya estuvo bonito de tanto dolor. (Twitter) 
(2) Concreta, manito, tú sabes, que arrastre y que agarre, uno que pegue y ya estuvo (CREA)

(3) Ya estuvo que no superaré esta canción. (Twitter)

Este estudio intenta mostrar, desde los postulados de la Gramática Cognoscitiva (Langacker, 1987: 11-430), la manera en que los rasgos nucleares de la construcción ya estuvo determinan la existencia de un conjunto de extensiones construccionales que comparten la noción de linde. Dada la naturaleza de la construcción, el estudio se centra exclusivamente en ejemplos del español mexicano. Proponemos que la construcción ya estuvo es la suma de los significados de sus componentes de base. A pesar de ser construccional, de su significado se pueden identificar los rasgos de límite o linde que aporta cada miembro de la construcción. Conviven en ella marcas que apuntan todas hacia la noción de límite: la telicidad verbal, la demarcación extrema con adverbios de gradación, la demarcación puntual por medio de preposiciones y el "anclaje conceptual" que impone la partícula ya (Maldonado y Delbecque 2009: 191; Delbecque y Maldonado 2011: 74). El alto nivel de lexicalización de ya estuvo como frase terminativa ha permitido que la construcción elida alguno de los elementos de cierre, sin que se pierda el significado de linde. De ahí que la forma más extendida de esta construcción sea aquella que presenta únicamente el verbo perfectivo junto con el anclaje ofrecido por ya: ya estuvo. De manera similar, la construcción presentativa ya estuvo que + oración completiva presenta y valora como pertinentes para el momento de la enunciación eventos que sucederán próximamente. En estos casos, el ancla ya valida la noción de fin de un evento como pertinente para el momento de la enunciación y lo acerca subjetivamente al conceptualizador. La notable relación entre las distintas construcciones ( $y a$ estuvo bueno, ya estuvo que, etc.) sugiere que los rasgos nucleares de la construcción ya estuvo determinan las extensiones construccionales que comparten las lecturas con valores terminativos o de suficiencia.

Palabras clave: telicidad, ancla, construcciones terminativas, metonimia sintáctica 


\section{INTRODUCCIÓN}

Notable, casi sorprendente, es el número de colocaciones lexicalizadas o semilexicalizadas que existen en el español de México con el polifacético adverbio ya: ya fue, ya mero, ya valió, desde ya, ya vas, ya mismo, etc. El presente trabajo intenta ahondar en el contenido de otra construcción de notable frecuencia: ya estuvo. Su significado cambia desde el canónico valor locativo hasta el de predicción, pasando por una gama de significados de sutil diferenciación: de su uso canónico como verbo locativo (1) se dan extensiones de logro o conclusión (2), suficiencia (3) y (4), o previsión o vaticinio (5) y (6):

(1) A ellos se les unirá el también delantero Carlos Sauri, quien ya estuvo en un entrenamiento de los Rayados hace dos meses. (Twitter)

(2) Dice además que está recopilando datos para un programa de radio con el que sí va a pegar pero todavía no le da forma "concreta, manito, tú sabes, que arrastre y que agarre, uno que pegue y ya estuvo". (CREA)

(3) Hoy termino con Cablevisión. Ya estuvo bonito de tanto dolor. (Twitter)

(4) Dígales a los que faltan que por este día ya estuvo bueno de audiencias. (CEMC)

(5) Si seguimos pensando que las vacas sagradas nos van salvar, pues ya estuvo que perdimos. (CREA)

(6) Ya estuvo que no superaré esta canción. (Twitter) 
El presente estudio intenta mostrar cómo de un significado de base se generan nuevas construcciones que, si bien responden al esquema original, son reelaboradas imponiendo nuevas conceptualizaciones. Proponemos, a lo largo de este estudio, que la construcción ya estuvo podría tener un significado unitario, con valor concatenativo de linde o límite de la predicación, cuya interpretación sería terminativa o de suficiencia. Sus extensiones dependerán de la pérdida de rasgos semánticos que se dé como consecuencia de su extensión a dominios cognoscitivos específicos. La hipótesis sugiere que los rasgos nucleares de la construcción ya estuvo determinan el tipo de extensiones construccionales que, más que presentar significados diferentes, comparten la noción de linde o cierre que se construye a partir de la suma de significados de sus componentes de base.

La muestra está conformada por 100 ejemplos del español mexicano, 8 corresponden al Corpus del Español Mexicano Contemporáneo (CEMC), 23 al Corpus de Referencia del Español Actual (CREA), 11 al Corpus Sociolingüístico de la Ciudad de México (CSCM), mientras que 58 provienen de la red social Twitter. El carácter informal de la construcción hace que se presente dominantemente en redes sociales.

El estudio parte de los postulados de la Gramática Cognoscitiva (Langacker 1987: 11-96, 1999:1-34) según la cual el significado está equiparado con la conceptualización. La lengua es entendida como un conjunto de unidades simbólicas de distintos niveles (sintácticas, semánticas y fonológicas) que están interiorizadas como patrones cognoscitivos interconectados en la mente del hablante. En consecuencia, no hay una división tajante entre los niveles semántico, pragmático y sintáctico de la lengua, sino que conforman un continuum de interacción múltiple. Esta interac- 
ción incluye la posibilidad de que unas construcciones influyan en la conformación de otras. Se postulan, entonces, relaciones metonímicas, en las que algunos de los rasgos de una construcción pueden heredarse en los de otras conformando redes semántico-sintácticas que reflejan el grado de motivación que se da dentro de la lengua (Flores y Maldonado 2016: 5).

El presente estudio intenta mostrar cómo de un significado de base se generan nuevas construcciones que, si bien responden al esquema original, son reelaboradas imponiendo nuevas conceptualizaciones. Para ello, partimos del principio de que la metonimia sintáctica es un proceso productivo en que unas construcciones influyen en otras al heredarles algunos de sus rasgos. En la medida en que una construcción presenta los rasgos de otra, podemos pensar en relaciones similares a las que se dan en la metonimia clásica entre el todo y la parte. Para dar cuenta de los distintos usos de ya estuvo expuestos en (1-6), será necesario examinar aquellas construcciones que preceden a la construcción que nos ocupa. Así, el estudio comienza por analizar aquellas que reúnen un verbo límite (estar) con preposición y adverbio resultativo y sus extensiones con adjetivos adverbializados. Ello permitirá identificar qué elementos de linde agrega el ancla ya cuando se suma a estas construcciones. Posteriormente se analiza ya estuvo, la más extendida de estas construcciones, que, preserva su significado de linde a pesar de ser la que menos marcas formales presenta. Su alto grado de lexicalización permite obviar alguno de sus componentes. Por último, se analiza una nueva extensión donde se suma a esta construcción una oración subordinada completiva que, una vez más, hereda el rasgo terminativo de la construcción de base. 
1. Significado de BASE

El verbo estar (del lat. stare, 'estar de pie'), al perder su contenido modal de verticalidad, continuó diferenciándose de ser por indicar una posición transitoria; este último valor fue, precisamente, el que condicionó su empleo como verbo copulativo y auxiliar (Cuervo 1994: 1097). Aceptemos, únicamente, estos contextos como los naturales del verbo estar: en (7) lo que se ofrece es una locación que equivaldría a hallarse en un lugar o situación; mientras que en (8) estaríamos frente al predicado atributivo que otorga al sujeto una cualidad o condición. Otro elemento importante de su significado es el aktionsart. Estar es un verbo de estado: estático, no dinámico, no télico, no puntual (Van Valin 2005: 33).

(7) Está en el concierto.

(8) Está interesante el concierto.

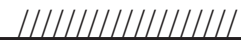

C/ME
Línea temporal y duración de la acción: /////

\section{Figura 1. Representación del verbo estar imperfectivo}

La Figura 1 muestra, justamente, cómo se representaría el verbo estar en los ejemplos (7) y (8). El evento coincide con la presencia del conceptualizador (C) o con el momento de enunciación (ME) y no tiene un fin delimitado. Sin embargo, cuando estar se vuelve perfectivo, algunos de sus rasgos cambian, como en (9): 
(9) Estuvo bonito el concierto.

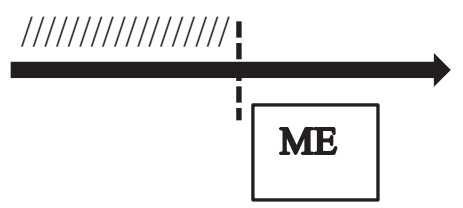

Línea temporal y duración de la acción: /////

Figura 2. Representación del verbo estar perfectivo

Como puede verse en la Figura 2, si se conjuga el verbo que era de estado en un tiempo perfectivo, como el pretérito simple, se vuelve verbo de realización (télico) y denota un proceso concluido, con límite marcado por el verbo. El evento se ve como ya concluido en el momento de la enunciación (ME).

\subsection{La frase construcción demarcativa ya estuvo}

Ahora bien, la frase ya estuvo parte de una construcción anterior o más bien conlleva una serie de elisiones que responden a los procesos propios de una construcción cuando se ha lexicalizado, a tal punto que permite la pérdida de algunos de sus elementos sin que ello cambie radicalmente su significado. Como habíamos establecido, partimos del principio según el cual el proceso de lexicalización de estas construcciones demuestra que su estructura semántica constituye la base para la formación de una serie de significados construccionales que compartirán rasgos comunes. Langacker (2009: 40.49) reconoce la metonimia en la sintaxis como un proceso productivo en la formación de significado, donde las propiedades 
de una construcción y otra se superponen para motivar una nueva construcción. Es decir, se establecen relaciones metonímicas en las que unas construcciones condicionan a las otras y van conformando una red con significados asociados. Sostendremos que para llegar a tener una construcción como (10) es necesario contar con construcciones más básicas como las de (11) y (12).

(10) Lluvia ¿por qué no regresas? Ya estuvo bueno de calor. (Twitter)

(11) Ay bájenle estuvo bien de tanta presunción, ya se parecen a los políticos presume y presume. (Twitter)

(12) Estuvo bueno de ser la gorda que se queda sentada en todas las bodas acabándose todo el alcohol. (Twitter)

Postulamos que la construcción que nos ocupa aglutina una serie de elementos que contribuyen, cada uno, a perfilar la noción de linde. Se reúnen en ella el verbo estar con marcación perfectiva, un adverbio resultativo (Kovacci 1999: 731), una preposición de o con (10) y un ancla conceptual ya (Maldonado y Delbecque 2009: 191) que, como se explicará más adelante, enmarca el evento en marcos contextuales específicos.

\subsection{Verbo limite con preposición y adverbio resultativo}

El hablante o conceptualizador al hacer télico el verbo de estado marca un límite, un final necesario del evento respecto de su perspectiva, en el momento de la enunciación. Las preposiciones que acompañan al verbo de realización (de/con) presuponen la existencia de una situación previa 
al verbo que opera en este ámbito como cierre. La ausencia de alguna de estas preposiciones sí implica la pérdida del cierre:

(13) Estuvo bueno el concierto (No se produce la lectura de límite de lo satisfactorio.)

Nótese además que en (13) bueno opera como adjetivo predicativo de concierto. En cambio, el empleo de un adverbio, comúnmente de modo resultativo (Kovacci 1999: 731), aparece en posición posverbal y modifica el verbo. La aparición de este tipo de adverbios como bueno o bien marca la idea de término de la acción, pero ahora califica el resultado dentro de una escala de lo satisfactorio.

(14) Estuvo bien de juerga me pondría mi madre a estas horas, acabamos por hoy. (Twitter)

(15) Bueno, ya para la casaa! Estuvo bueno de paseo. (Twitter)

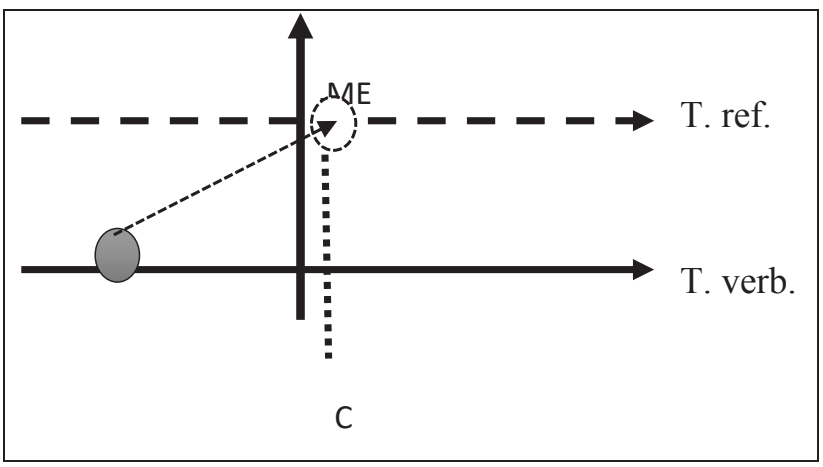

\section{Leyenda:}

ME: Momento de la enunciación

C: Conceptualizador

T. ref.: Tiempo

referencial

T. verb.: Tiempo verbal

Figura 3. Representación del verbo con respecto al momento de la enunciación 
La Figura 3 representa cómo el verbo, que tiene una marca morfológica de pretérito (el círculo continuo en la flecha continua del tiempo verbal), pero que no referencia a un evento del pasado, es traído por el conceptualizador (C) al momento de la enunciación (ME) (con el círculo punteado) (ver ejemplos 14 y 15). Es decir, el tiempo referencial y el tiempo morfológico verbal no coinciden y esto provee un valor terminativo a la construcción que se reafirma con la presencia de la preposición y el adverbio.

La construcción "prototípica", o al menos la más extendida en el uso, solo admite adverbios de modo (resultativos). Los adverbios de lugar, de cantidad, de afirmación, de tiempo, etcétera, no promueven la noción de gradación que sí ofrecen los resultativos. Otro tipo de adverbios no licencian la noción de cierre y son inadecuados para este tipo de construcción.

(16) Dígales a los que faltan que por este día estuvo bien de audiencias. (Twitter)

* Dígales a los que faltan que por este día estuvo tarde de audiencias.

(17) Estuvo bueno de soportar a este pernicioso calumniador. (Twitter)

* Estuvo mucho de soportar a este pernicioso calumniador.

2. EXTENSiONes de uso de la CONSTRUCCión. Adjetivos adVerbializados

Dentro de las extensiones de esta construcción es posible encontrar ciertas variaciones que se estructuran sobre la misma base. En lugar de 
emplear un adverbio, la noción de límite se marca a través de un adjetivo adverbial (Hummel 2013: 218). Pero, al igual que con los primeros, no cualquier tipo de adjetivo puede ser empleado (18):

(18) Pos que ya estuvo suave ¿no? Que aquí se rompió una taza y que cada quien jala para su casa. (CREA)

Los adjetivos son adverbializados en virtud de que tienen alcance verbal y carecen de marcas de femenino y de plural. Sólo es posible extender la construcción con el empleo de adjetivos que, al igual que los adverbios resultativos, expresen la evaluación gradual de algo (chido, sabroso, suave, chingón, etc.)

(19) Ya estuvo bonito de cantar con fiebre. (Twitter)

(20) Kongregate, ya estuvo bonito con los jueguitos de bicis, primer aviso. (Twitter)

(21) Dijera Ninel (ella, muy sabia) ya estuvo sabroso de tanta rezadera. Ahora a lo que venimos, ¡a pecar! (Twitter)

Cada uno de estos adjetivos adverbializados, al unirse al verbo de límite, muestra una extensión de su significado que será opuesto a lo que normalmente implica. Por ejemplo, el adjetivo suave (el más frecuente de todos los encontrados), en la construcción implica que se ha perdido la suavidad y ha operado un cambio de estado que ha llegado a su límite satisfactorio y que debe ser invertido. 
(22) "Bueno, Molkas, para broma ya estuvo suave", dijo Palinuro, amenazando con darle una cachetada. (CREA)

(23) Pero no quiero terminar como ayer jajaja ya estuvo suave de champagne. (Twitter)

$\mathrm{Al}$ igual que con los casos anteriores, hay una implicación de cierre del estado previo al momento de la enunciación y el adjetivo adverbializado la reafirma al calificar su resultado. El empleo de otros adjetivos adverbiales no canónicos en la construcción requiere tanto la presencia explícita de ya, como de la frase preposicional que especifique el ámbito en que el linde es alcanzado. La ausencia de estas especificaciones de linde cancela la lectura de linde, como en (24b) o hacen la expresión cuestionable, como en (24c).

(24) a. Kongregate, ya estuvo bonito con los jueguitos de bicis, primer aviso. (Twitter)

b. Kongregate, estuvo bonito con los jueguitos de bicis, primer aviso. (Sin ancla ya)

c. \#Kongregate, estuvo bonito los jueguitos de bicis, primer aviso. (Sin preposición)

\subsection{La demarcación del ancla ya}

Desde la Gramática Cognoscitiva, toda ancla es una marca gramatical que se encarga de ubicar el evento en un contexto particular que el hablante ubica en el momento de la enunciación. Para el sustantivo, los artículos y los demostrativos son anclas fundamentales, mientras que 
para el verbo lo son las marcas de tiempo y persona. Las anclas incluyen el contorno pragmático que resulta relevante para los participantes del intercambio comunicativo.

$\mathrm{Ya}$ es una predicación de anclaje que permite a los participantes del discurso delimitar un elemento específico perteneciente a un marco conceptual compartido tanto por el hablante como por el oyente (Maldonado y Delbecque 2009: 191; Delbecque y Maldonado 2011: 74). En el caso que nos ocupa, ya ubica el evento en el espacio discursivo y lo valida según las circunstancias de enunciación. Su significado es puntual y su valor proyectivo; es decir, anticipa ocurrencias predecibles y permite ver algo como terminado cuando aún no ha concluido (\$Figura 4).

(25) Lluvia ¿por qué no regresas? Ya estuvo bueno de calor. (Twitter)

(26) Pos que ya estuvo suave ¿̨no? Que aquí se rompió una taza y que cada quien jala para su casa. (CREA)

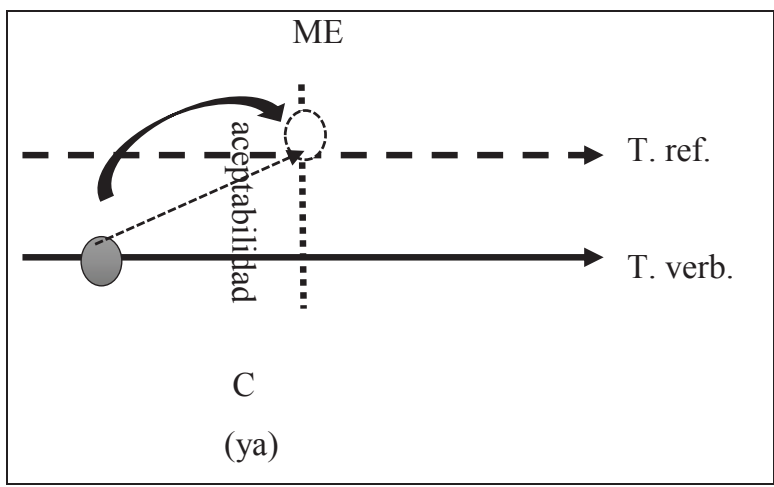

\section{Leyenda:}

ME: Momento de la enunciación

C: Conceptualizador

T. ref.: Tiempo referencial

T. verb.: Tiempo verbal

Figura 4. Representación de la acción de ya sobre el verbo y con respecto al momento de la enunciación 
En la Figura 4 se muestra, una vez más, un evento morfológicamente marcado en pasado, pero con una referencia temporal que coincide con el momento de la enunciación. Así mismo, se señala cómo ya marca los lindes de un hecho desde la perspectiva del momento del habla. Como lo indica la flecha curva, con ya el punto final del evento no concluido se proyecta al momento de la enunciación como un deseo del hablante de que dicho evento llegue a su conclusión y esta noción de cierre se ve reforzada con la presencia de la preposición y el adjetivo adverbializado.

\section{Degradación de la construcción (elisiones de lindes)}

En la construcción demarcativa ya + verbo télico + adv./adj. resultativo + preposición se suman distintos elementos de linde (telicidad, adverbios $\mathrm{o}$ adjetivos de gradación, ya). Mientras más elementos de cierre o límite sean empleados por el conceptualizador más se refuerza la idea de un cambio de estado requerido, según el grado de suficiencia de lo que es satisfactorio para el hablante. No obstante, la modalidad más extendida de esta construcción no es aquella que tiene explícitos todos sus elementos; por el contrario, es la más degradada la que se ha generalizado. Su avanzado proceso de lexicalización es el que licencia la pérdida de elementos sin que se afecte su significado. Existe así en su uso la "posibilidad" de reducir o escoger la cantidad de lindes que se emplean. Por una parte, es posible formular enunciados demarcativos sin adverbios resultativos (27a) o sin adjetivos adverbializados (28a), pero, en esos casos, el ancla ya sí debe estar presente para trasmitir el sentido de límite (27b) y (28b). 
(27) a. ¡Bueno, ya estuvo, vamos a echarnos el disparejo! (Twitter)

b. ${ }^{*}$ ¡Bueno estuvo!, vamos a echarnos el disparejo.

(28) a. Si el chiste nada más era "por ahí pasé" ipues ya pasó! ¡y ya vámonos ya a volar! Ya estuvo. (Twitter)

b. * Si el chiste nada más era "por ahí pasé” ¡pues ya pasó! ¡y ya vámonos ya a volar! Estuvo.

Por otra parte, también tenemos la posibilidad de producir estos enunciados sin el ancla $y a$, como en (29a) y (30a), pero en este caso se vuelve imprescindible la presencia tanto del adverbio resultativo, como de la preposición. Si alguno de estos elementos faltara, tampoco se podría recuperar la lectura de linde (29b) y (30b).

(29) a. Bueno, estuvo bien de pelear.

b. * Bueno, estuvo de pelear.

(30) a. Estuvo bien de burlas, también hay que entender su punto de vista.

b. *Estuvo de burlas, también hay que entender su punto de vista.

En los casos perfectivos, la noción de límite se mantiene, pero con ciertas variaciones de significado: en los ejemplos (27) y (28), a pesar de elidirse el adverbio, resulta totalmente recuperable la noción de límite dejada por su huella. Los ejemplos (29) y (30), que carecen del ancla $y a$, si bien mantienen la noción de cierre (otorgada por el adverbio, por la telicidad del verbo y por la preposición), son menos frecuentes en el uso. Esto puede deberse a la doble función desempeñada por el ancla ya, 
la de límite, por una parte, y la de actualización de los elementos pertinentes para el momento de la enunciación, cosa que resulta necesaria para el cambio que el hablante quiere hacer notar.

Como es de esperar, la marca de telicidad es requisito indispensable para la construcción. Todos los ejemplos encontrados presentan el verbo estar en perfectivo. Bien es cierto que, si están presentes los demás demarcadores, en la construcción demarcativa se puede emplear estar en presente a fin de hacer performativos demarcativos, como en (28c) y (30c):

(29) c. Bueno, ya está bien de pelear.

(30) c. Ya está bien de burlas, también hay que entender su punto de vista.

En presente se imponen la suspensión inmediata de la acción que designa el verbo principal, según el evento está ligado con el momento de enunciación.

4. Una NUEVA EXTENSIÓN. Verbo LÍMite y ORACiONES SUbordinadaS

Otra posible extensión de la construcción de límite es aquella que está introducida por la conjunción subordinante que, encargada de introducir una completiva de sujeto. Se trata de una construcción presentativa que anticipa el cumplimiento de un evento en el nuevo enunciado; el verbo mantiene su implicación de linde, pero en estos casos la extiende hasta la frase completiva y promueve, una vez más, la predicción de la 
terminación del evento, como en (31) y (32). Nótese que se trata de un evento marcado en futuro cuya terminación es predicha:

(31) Bueno, estuvo que Jimmy Morales no se prestará a su circo. (Twitter)

(32) No pues ya estuvo que no dejaré en blanco mi próximo examen porque es oral. ... (Twitter)

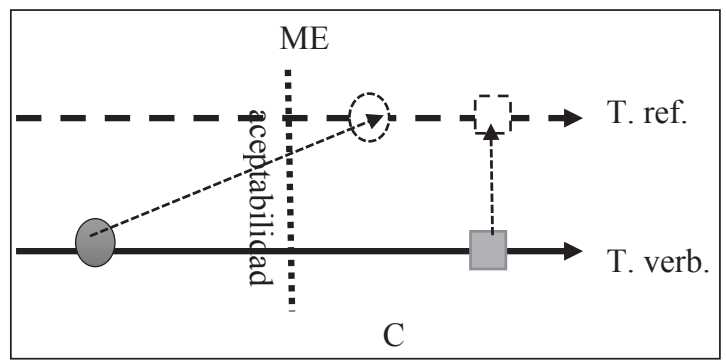

\section{Leyenda:}

ME: Momento de la enunciación

C: Conceptualizador

T. ref.: Tiempo referencial

T. verb.: Tiempo verbal

Figura 5. Representación de los verbos con respecto al momento de la enunciación

La Figura 5 esboza la estructura de la construcción. El evento no acontecido, marcado en futuro (el cuadrado punteado), se da por hecho en el momento de la enunciación con un valor terminativo (la flecha punteada que liga al cuadrado con el círculo sombreado antes del momento de la enunciación). Si bien el verbo estar perfectivo es suficiente para marcar el linde de la predicación de la oración completiva, ya intensifica la perspectiva del hablante (cambio del estado anterior) y, en combinación con el tiempo verbal de la frase subordinada, presenta y valora como pertinentes para el momento de la enunciación eventos que terminarán próximamente. Todo esto evidencia una vez más su significado fundamentalmente proyectivo. 
(33) ¿Cómo Sophia cupo ahí? si yo apenas meto un pie y ya estuvo que rompí toda la bolsa (Twitter)

(34) Ya estuvo que no fui a trabajar mañana (Twitter)

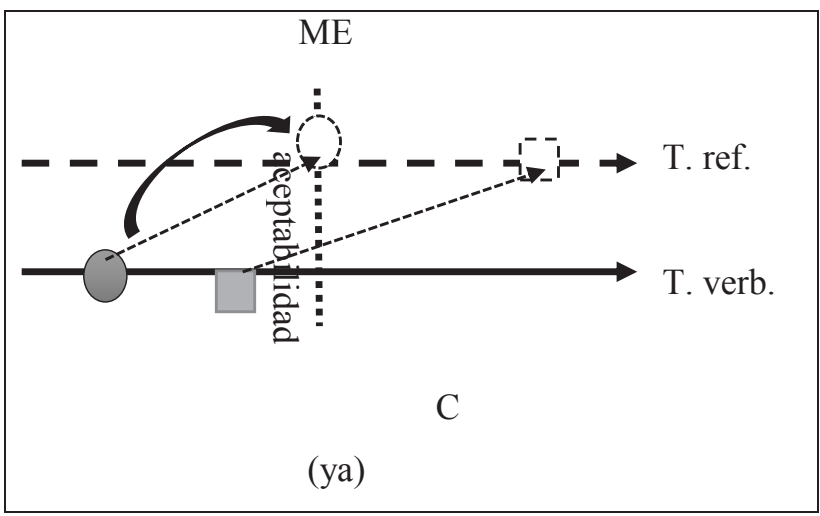

Leyenda:

ME: Momento de la enunciación

C: Conceptualizador

T. ref.: Tiempo referencial

T. verb.: Tiempo verbal

Flecha curva: límite de la acción

Figura 6. Representación de la acción de ya sobre el verbo y con respecto al momento de la enunciación

La Figura 6 describe lo que sucede en ejemplos como (33) y (34). La presencia de $y a$, además de puntualizar la inminencia de los sucesos, posiciona el nuevo evento morfológicamente construido en pasado como posterior al momento de la enunciación, por lo que se asume con alto nivel de certeza como un hecho que sucederá. $Y a$ abre un acceso al acto futuro conceptualizado por el hablante y expresado como pasado. Además, hace posible un acercamiento subjetivo del evento al momento de la enunciación: al emplear el futuro, el evento es más distante (menos real), mientras que el uso del pretérito implica mayor grado de certeza o seguridad respecto de su acaecer; pero ambas cons- 
trucciones poseen el mismo valor terminativo, aunque, quizás, con un nivel diferente. Mientras el primero impone la terminación de un evento en la realidad del hablante, el segundo confiere a esa terminación un carácter predictivo.

\section{Conclusiones}

La frase ya estuvo es una construcción en que se suman, por lo menos, dos marcas de linde: telicidad temporal, una preposición puntual o una predicación de anclaje ya, que, al presentarse juntas, refuerzan la noción de límite que cada una de ellas impone por separado. Esta construcción puede ser reforzada por los adverbios de grado que toman valor puntual extremo en la construcción. Su nivel de lexicalización como frase terminativa ha permitido que se elida alguno de sus elementos de linde, sin que se pierda ese significado. De ahí que la forma más extendida de esta construcción sea aquella que presenta únicamente el verbo perfectivo junto con la preposición y el anclaje ofrecido por ya: ya estuvo.

Por otra parte, la construcción presentativa ya estuvo que + oración completiva también presenta y valora como pertinentes para el momento de la enunciación eventos que sucederán próximamente. En estos casos, el ancla $y a$, además de aportar la noción de fin a un evento situado en la anterioridad o su proyección hacia el futuro, lo valida como pertinente para el momento de la enunciación y lo acerca subjetivamente al conceptualizador.

La íntima relación entre las distintas construcciones sugiere que los rasgos nucleares de la construcción ya estuvo, en que se suman perfiles 
de linde, determinan las extensiones construccionales que comparten las lecturas con valores terminativos o de suficiencia.

$\mathrm{Al}$ esquematizar las construcciones analizadas es evidente que unas construcciones condicionan a las otras y van conformando una red con significados asociados.

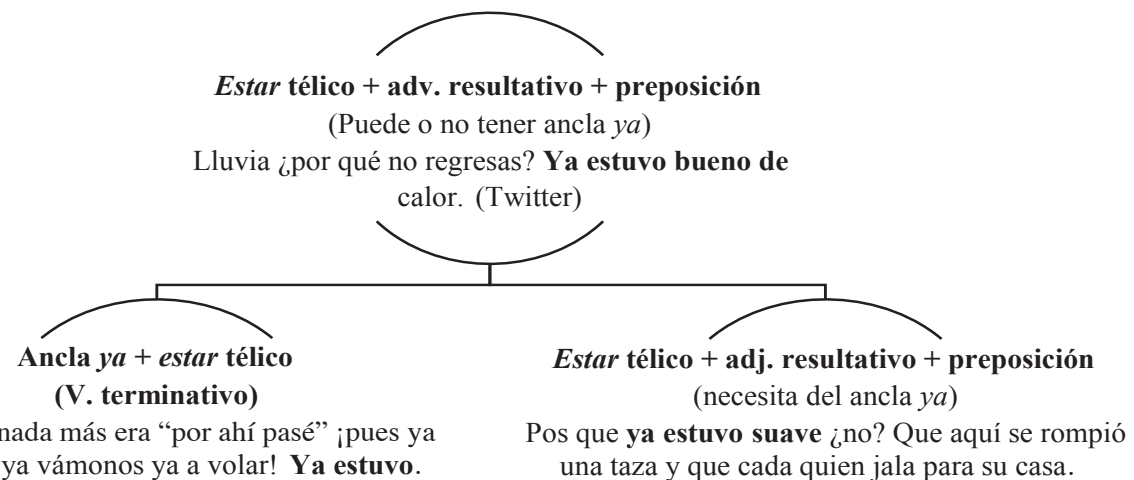

Si el chiste nada más era "por ahí pasé" ¡pues ya
pasó! ¡y ya vámonos ya a volar! Ya estuvo.

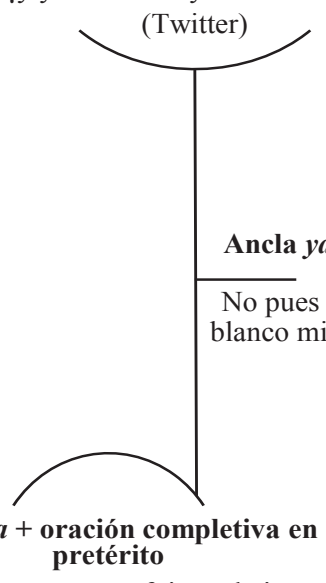

Ya estuvo que no fui a trabajar mañana. (Twitter)

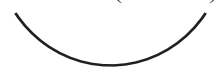

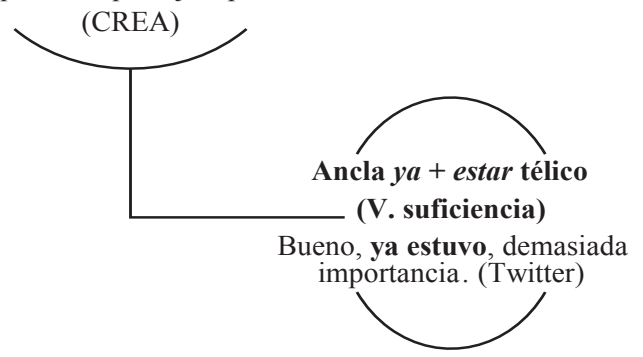

Figura 7. Red de significados asociados a la construcción ya estuvo 
La red en la Figura 7, muestra las interconexiones entre la construcción de base (ya) estuvo bien de y sus posibles realizaciones. La relación existente entre estas construcciones responde a los procesos metonímicos que permiten que una construcción adquiera rasgos de la anterior y los herede a otras más. De la construcción de base Estar télico + adv. resultativo + preposición se desprenden dos posibles realizaciones, una en que demarca suficiencia asociada a un límite, heredado del adverbio resultativo, ahora convertido en adjetivo (Estar télico + adj. resultativo + preposición Pos que ya estuvo suave) y otra que especifica el dominio respecto del cual dicho límite se impone (Ancla ya + estar télico: ya estuvo: iy ya vámonos ya a volar! Ya estuvo). De este segundo nivel se desprenden, a su vez, dos construcciones completivas introducidas por la conjunción subordinante que, que anticipan el cumplimiento de un evento en el nuevo enunciado, ya sea con un evento no acontecido (Ancla ya + oración completiva en futuro), marcado en un futuro pero que se da por hecho en el momento de la enunciación con un valor terminativo; o mediante el pretérito visto como posterior al momento de la enunciación (Ancla ya + oración completiva en pretérito) que se asume con alto nivel de certeza como un hecho que sucederá.

Fuentes DE DATOS:

Corpus del Español Mexicano Contemporáneo (CEMC), <http://www. corpus.unam.mx/cemc>, software AMATE ver 1.0. (Consultado en octubre de 2015). 
Corpus del Proyecto para el Estudio Sociolingüístico del Español de España y de América, (PRESEEA), <http://preseea.linguas.net>. (Consultado en octubre de 2015).

Corpus de Referencia del Español actual (CREA), <http://www.rae.es >. (Consultado en octubre de 2015).

Twitter, <https://twitter.com/?lang=es>. (Consultado en octubre de 2015).

\section{REFERENCIAS}

Academia Mexicana de la Lengua. 2010. Diccionario de mexicanismos. México DF: Siglo XXI.

Cuervo, Rufino José. 1994. Diccionario de construcción y régimen de la lengua castellana. Santa Fe de Bogotá: Instituto Caro y Cuervo.

Delbecque, N., y Maldonado, R. 2011. Spanish ya. A conceptual pragmatic anchor. Journal of Pragmatics, 43(1), 73-98.

Flores Cervantes, Marcela, y Maldonado Soto, Ricardo. 2016. Metonimia sintáctica en construcciones de transferencia. Nueva Revista de Filología Hispánica, 64(1). 1-26.

García Fernández, Luis. 1999. Los complementos adverbiales temporales. La subordinación temporal. En V. Demonte, I. Bosque (coords.), Gramática descriptiva de la lengua española, 3129-3208. Madrid: Espasa Calpe.

Hummel, Martin. 2013. Sincronía y diacronía de los llamados adjetivos adverbializados y de los adverbios en -mente. Anuario de Letras: Lingüistica y Filología, 1-2. 215-282. 
Kovacci, O. 1999. El adverbio. En V. Demonte, I. Bosque (dir.), Gramática descriptiva de la lengua española, 706-786. Madrid: Espasa Calpe. Langacker, Ronald W. 1999. Grammar and conceptualization. Berlín: Walter de Gruyter.

Langacker, Ronald W. 1987. Foundations of Cognitive Grammar, Vol. 1 Theoretical Prerequisites. Stanford: Stanford University Press.

Langacker, Ronald W. 2009. Metonymy in Grammar. Investigations in Cognitive Grammar. Cognitive Linguistics research 42. Berlín: Mouton de Gruyter. 40-59.

Maldonado, Ricardo y Delbecque, Nicole. 2009. Ya, ancla conceptual de una visión programática. En L. Puig (ed.), El discurso y sus espejos, 189-235. México: Universidad Nacional Autónoma de México. Torner Castells, Sergi. 2005. Aspectos de la semántica de los adverbios de modo en español. Barcelona: Universitat Pompeu Fabra. (Tesis doctoral).

Van Valin, Robert D. 2005. Exploring the Syntax-Semantics Interface. Cambridge: Cambridge University Press. 